\title{
Patient Perspectives on Health-Related Quality of Life in Diffuse Large B-Cell Lymphoma Treated with Car T-Cell Therapy: A Qualitative Study
}

\author{
Rebecca Cheng · Kayla Scippa · Frederick L. Locke · Julia Thornton Snider • \\ Heather Jim
}

Received: July 6, 2021 / Accepted: October 5, 2021 / Published online: November 15, 2021

(C) The Author(s) 2021

\section{ABSTRACT}

Introduction: Chimeric antigen receptor T-cell (CAR T) therapy offers a potentially curative option for patients with relapsed and refractory hematologic malignancies, including diffuse large B-cell lymphoma (DLBCL). Patient-reported experiences with CAR $\mathrm{T}$ therapy are limited and have not been well characterized. The purpose of this qualitative study was to explore patient descriptions of key domains of health-related quality of life (HRQoL) in DLBCL patients treated with CAR T therapy.

Methods: A targeted literature review was initially conducted to inform the development of the interview guide comprising predetermined open-ended questions. Two focus groups were conducted with a total of 18 patients with DLBCL identified from patient advisory boards. Focus group sessions were recorded and transcribed verbatim. MAXQDA 18.2.0 qualitative

R. Cheng ( $\square)$

ZS Associates, Thousand Oaks, CA, USA

e-mail: otmanuscript@gmail.com

K. Scippa

ZS Associates, Princeton, NJ, USA

F. L. Locke · H. Jim

H. Lee Moffitt Cancer Center and Research Institute, Tampa, FL, USA

J. T. Snider

Kite, A Gilead Company, Santa Monica, CA, USA data analysis software was utilized to facilitate a constant-comparative coding process to identify key concepts.

Results: Eight domain impairments (social functioning, emotional functioning, fatigue, physical functioning, cognitive functioning, role functioning, sleep, and pain/discomfort) were identified from the qualitative analysis and endorsed by DLBCL patients treated with CAR T. Compared with before CAR $\mathrm{T}$ therapy, patients reported increased impairment in every domain during or immediately after CAR T therapy. This impairment improved for each domain 6 months after CAR T therapy except for pain/discomfort. Compared with before CAR $\mathrm{T}$ therapy, improvement in impairment for each domain was observed 6 months after CAR T therapy except for fatigue, sleep, and pain/ discomfort.

Conclusion: This study provides meaningful information regarding the impact of CAR $\mathrm{T}$ therapy on HRQoL in patients with DLBCL throughout their treatment journey. Health care professionals and investigators can utilize these data in examining existing patient-reported outcome (PRO) measures that are used in DLBCL clinical trials and to better understand the needs of DLBCL survivors.

Keywords: Chimeric antigen receptor (CAR) T-cell therapy; Diffuse large B-cell lymphoma; DLBCL; Health-related quality of life; HRQoL; 
Non-Hodgkin lymphoma; Patient-reported outcome

\section{Key Summary Points}

Chimeric antigen receptor (CAR) T-cell therapy offers a potentially curative option for patients with relapsed and refractory hematologic malignancies, including diffuse large B-cell lymphoma (DLBCL).

Patient-reported experiences with CAR T therapy are limited and have not been well characterized.

The purpose of this qualitative study was to explore patient descriptions of key domains of health-related quality of life in DLBCL patients treated with CAR T therapy.

The use of patient-reported outcomes (PRO) measures, specifically key domains identified in the study, may help clinicians better understand the patient needs for this disease state and reinforce therapeutic approaches.

PROs provide qualitative information on different aspects of their disease before, during, and after treatment to track their journey.

These data can be used to help inform programs that may provide better support to DLBCL patients and caregivers.

\section{INTRODUCTION}

Diffuse large B-cell lymphoma (DLBCL) is the most common form of non-Hodgkin lymphoma (NHL), representing approximately 30\% of all NHL cases in the United States, with an incidence rate of 5 per 100,000 people and a mortality rate of 1 per 100,000 people per year. Individuals who are middle-aged or elderly, male, or Caucasian are at higher risk of developing DLBCL, and morbidity and mortality are especially significant in the relapsed/refractory $(\mathrm{R} / \mathrm{R})$ patient population [1-4]. Common symptoms are nonspecific and typically include swollen lymph nodes, weight loss, and fever or chills [7]. The diagnosis is made by histologic evaluation of tumor biopsy, and prognosis can be informed by symptoms and radiologic/pathologic assessments [3-6].

The current standard of care (SOC) for DLBCL is chemoimmunotherapy with R-CHOP (rituximab-cyclophosphamide, hydroxydaunomycin, oncovin, and prednisone), which is effective in approximately half of the patients [8]. Patients with disease relapse or nonresponse to first-line treatment are often given salvage high-dose chemotherapy followed by autologous hematopoietic stem cell transplantation (HSCT) and may have poor outcomes and high unmet needs [3, 5, 9-11]. Patients who do not respond to second-line salvage chemoimmunotherapy, or who relapse after transplantation, have especially poor prognosis [12-14]. Recent US Food and Drug Administration (FDA) and European Medicines Agency (EMA) approvals of chimeric antigen receptor T-cell (CAR T) therapy options for R/R DLBCL, axicabtagene ciloleucel and tisagenlecleucel, as well as the recent FDA approval of lisocabtagene maraleucel, have provided a potentially curative option for patients with the worst prognosis [15-18].

While the primary focus of oncology treatment is on overall survival and progression-free survival (PFS), patient-reported outcomes (PROs) have become increasingly important in differentiating treatment options, especially for conditions in which refractory disease is common and/or PFS is short [19-22]. A PRO is "any report of the status of a patient's health condition that comes directly from the patient, without interpretation of the patient' response by a clinician or anyone else" [23]. In clinical trials, a PRO can be used as a tool to measure a patient's symptoms, signs, or an aspect of functioning directly related to disease status. PRO measures often represent the effect of disease on health-related quality of life (HRQoL) from the patient perspective [23]. Studies have demonstrated that $\mathrm{R} / \mathrm{R}$ DLBCL patients 
receiving SOC therapy report decreases in HRQoL across numerous domains including physical functioning and emotional well-being [24-26]. Similarly, CAR T therapy is associated with potentially severe adverse events, including cytokine release syndrome (CRS) and immune effector cell-associated neurotoxicity syndrome (ICANS), which can significantly impair patient functioning and HRQOL $[27,28]$.

PROs have been widely recognized as the best way to capture the patient perspective in oncology $[20,21]$ and have also garnered support from regulatory agencies [23, 29, 30]. In June 2020, the FDA issued final guidance on patient-focused drug development, which states that patients are best positioned to provide their experiences with respect to the impact of their condition and treatment over time [31]. Consequently, clinical trials are increasingly expected to incorporate the patient perspective through PROs as an important complement to clinical outcomes [21].

Despite the importance and regulatory endorsement of PROs, there is limited literature on the qualitative experiences of $\mathrm{R} / \mathrm{R}$ DLBCL patients, and even fewer PRO-related publications for R/R DLBCL patients treated with CAR T. Frequently used oncology-specific PROs include the EORTC QLQ-C30 (European Organisation for Research and Treatment of Cancer Quality of Life Questionnaire-Core 30) [32], FACT-LYM (Functional Assessment of Cancer Therapy-Lymphoma)[33], and FACT-G (Functional Assessment of Cancer Therapy-General) [26]. However, only the FACTLYM and FACT-G have been validated for the NHL population, but these instruments have not been specifically assessed in DLBCL patients treated with CAR T therapy $[33,34]$. The current study aimed to understand the overall treatment experience and journey of DLBCL patients treated with CAR $\mathrm{T}$ therapy and to evaluate domain-level coverage of the PROs commonly used to assess HRQoL in this population.

\section{METHODS}

\section{Targeted Literature Review}

A targeted literature review was conducted to inform the development of the focus group moderator guide. This review was conducted in May 2019 to identify publications in which PROs were used in the DLBCL patient population treated with CAR T therapy. While an a priori list was compiled before the search, every effort was made to identify any additional PROs to be included. The search was conducted in ClinicalTrials.gov, PubMed, the American Society of Clinical Oncology (ASCO) and the International Society for Pharmacoeconomics and Outcomes Research (ISPOR), currently the Professional Society for Health Economics and Outcomes Research, databases using search terms including "CAR T," "DLBCL," and "Patient-Reported Outcome" or "PRO," and limiting results to the last 10 years. The search in ClinicalTrials.gov yielded nine studies (citing the EQ-5D, EORTC QLQ-C30, PROMIS-29, FACT-G, and FACT-LYM) [32-38], and that in ASCO and ISPOR yielded three studies (citing the FACT-G). Corroborating the systematic literature review and meta-analysis conducted by Grigor et al. [39], no studies related to CAR T therapy that investigated PROs in DLBCL patients were identified from the PubMed search. Additional validated PROs of interest not identified in the literature search, such as the WPAI (Work Productivity and Activity Impairment) and MFI (Multidimensional Fatigue Inventory), were hand-selected by study investigators. These PROs were included in a comprehensive listing along with the PROs identified from the targeted literature review, and the domains for each PRO were evaluated (Table 1) and used to inform the discussion guide. Additional details on the included PRO instruments are provided in the Appendix.

\section{Participant Recruitment}

A convenience sample of patients with DLBCL treated with CAR $T$ therapy was recruited from industry-sponsored patient advisory board 
panels and invited to participate in an optional focus group at the conclusion of the panel. Invitation letters to participate in a focus group were sent to all 21 patient advisory board participants; $86 \%(n=18)$ of the patients accepted the invitation and were included in the focus groups. All patients provided written informed consent and were able to read, write, and comprehend English; had a physician-confirmed diagnosis of DLBCL; and received CAR T therapy. Patients were excluded from participation in the focus group if they were $<18$ years of age and/or did not speak English. Additionally, moderators may have disqualified a patient if there were concerns about his/her cognitive ability to complete the focus group session. Two focus groups were planned with a goal of 6-8 patients per group with representing various geographical regions in the US.

The qualitative study, titled "Qualitative Focus Groups Focusing on Symptoms and Impact on Patients with Diffuse Large B-cell Lymphoma (DLBCL) Treated with CAR-T," was submitted to the New England Independent Review Board (IRB) for ethics review and was approved on November 7, 2019, prior to any contact with focus group participants. The submission package (IRB\#120190471) to the IRB included the protocol, moderator guide, and informed consent. The focus group was approved to take place at the Grand Hyatt DFW, 2337 S International Pkwy, Dallas, Texas, 75261; however, due to the COVID-19 pandemic, a change in research protocol was submitted and approved on July 1, 2020, to conduct the second focus group virtually using a password-protected video conferencing platform. Video-recording via the video conferencing platform had also been approved. The authors have read and understood the declaration intended by the Declaration of Helsinki as a statement of ethical principles for medical researching involving human subjects, including research on identifiable human data. This study was performed in accordance with the Helsinki Declaration of 1964 and its later amendments.

\section{Data Collection}

The first focus group was conducted live at a hotel conference room in November 2019. It was audio recorded and lasted approximately 90 minutes. The second focus group was conducted virtually in July 2020 due to the COVID-19 pandemic and related statewide variances in reopening plans and travel restrictions. It lasted approximately 2 hours and was conducted via Zoom ${ }^{\circledR}$ (Zoom Video Communications Inc., 2020), an online videoconferencing platform that allowed for video and audio recordings. In both instances, each patient was compensated $\$ 75$ per hour of focus group participation.

The focus group format included concept confirmation and elicitation. The objective of the concept confirmation was to assess the relevance of the domains from the PROs identified, and the aim of the concept elicitation was to uncover additional unaddressed or underaddressed symptoms and disease-related themes for the patients. The PRO domains from the targeted literature review were assessed and translated and incorporated into easily understood questions for the focus group moderator guide. These questions were designed to elicit discussion and to ultimately capture relevant patient experiences before, during, and immediately after the CAR $\mathrm{T}$ procedure, and at 6 months after CAR T. The questions incorporated patient-friendly language and comprehensively covered the specific domains for the PROs previously identified to assess whether these domains were relevant and appropriate to this patient population, also known as concept confirmation in qualitative research. Concept elicitation was conducted where a series of questions were included to probe for any gaps that had not been covered. In addition to exploring caregiver impact, these questions probed on symptoms that were not previously covered such as headache, severe flu, delirium, or seizures, as well as any other burden or impact on quality of life as experienced by the patient. Each focus group was conducted by experienced facilitators using a moderator discussion guide. The moderator guide detailed the session format and content, a review of existing 


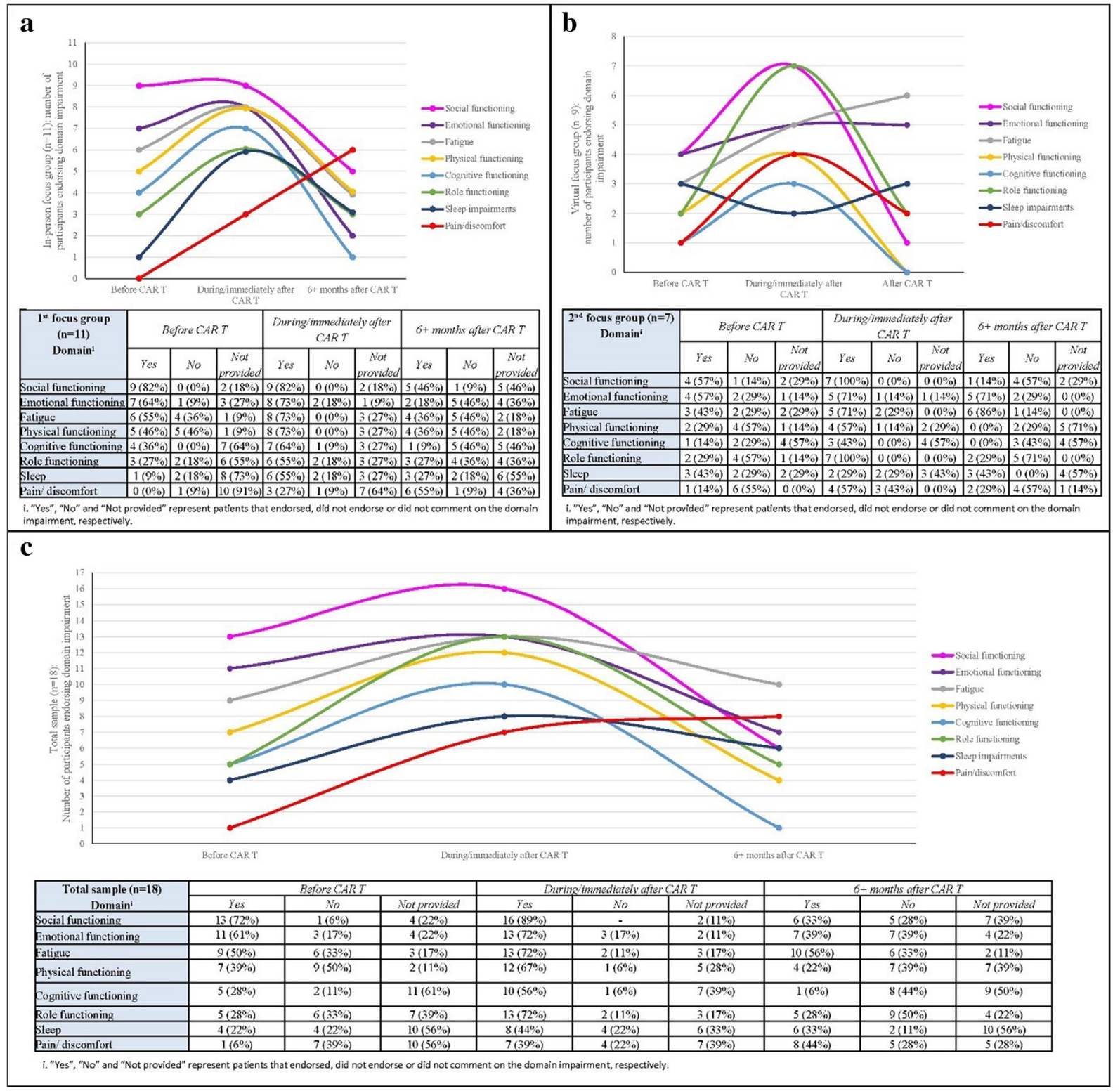

Fig. 1 HRQoL domain impairment frequency of experience before, during, and after CAR $\mathrm{T}$ therapy for a inperson focus group, $\mathbf{b}$ virtual focus group, and $\mathbf{c}$ total sample. Eight main domain impairments were identified in the qualitative analysis: social functioning, emotional functioning, fatigue, physical functioning, cognitive functioning, role functioning, sleep, and pain/discomfort.

PRO domains identified from the targeted literature review, and a general discussion of patients' HRQoL and burden of illness.
Patients highlighted impairments in social functioning, emotional functioning, fatigue, and role functioning when discussing experiences following CAR $T$ treatment. Following the patient journey from before CAR $T$ therapy to during or immediately after it and at 6 or more months after therapy, most domains showed an improvement, except for pain

\section{Data Analysis}

Audio recordings of the focus group sessions were transcribed verbatim and anonymized. A 
Table 1 Thematic overlap among patient-reported outcome measurements to inform development of moderator guide

\begin{tabular}{|c|c|c|c|c|c|c|c|}
\hline \multirow[t]{2}{*}{ Domain } & \multicolumn{7}{|c|}{ PRO measure } \\
\hline & $\begin{array}{l}\text { EQ-5D } \\
{[36]}\end{array}$ & $\begin{array}{l}\text { EORTC QLQ- } \\
\text { C30 [32] }\end{array}$ & $\begin{array}{l}\text { WPAI } \\
{[38]}\end{array}$ & $\begin{array}{l}\text { PROMIS- } \\
29[37]\end{array}$ & $\begin{array}{l}\text { MFI } \\
{[35]}\end{array}$ & $\begin{array}{l}\text { FACT-G } \\
{[33]}\end{array}$ & $\begin{array}{l}\text { FACT- } \\
\text { LYM [34] }\end{array}$ \\
\hline Physical functioning & $\mathrm{x}$ & $\mathrm{x}$ & & $\mathrm{x}$ & & $\mathrm{x}$ & $\mathrm{x}$ \\
\hline Emotional functioning & $\mathrm{x}$ & $\mathrm{x}$ & & $\mathrm{x}$ & & $\mathrm{x}$ & $\mathrm{x}$ \\
\hline Fatigue & & $\mathrm{x}$ & & & $\mathrm{x}$ & $\mathrm{x}$ & $\mathrm{x}$ \\
\hline Pain/discomfort & $\mathrm{x}$ & $\mathrm{x}$ & & $\mathrm{x}$ & & $\mathrm{x}$ & $\mathrm{x}$ \\
\hline Social well-being & & $\mathrm{x}$ & & $\mathrm{x}$ & & $\mathrm{x}$ & $\mathrm{x}$ \\
\hline Sleep & & & & $\mathrm{x}$ & & & \\
\hline Cognitive functioning & & $\mathrm{x}$ & & & & & \\
\hline Role functioning & & $\mathrm{x}$ & $\mathrm{x}$ & & & & \\
\hline Lymphoma-specific symptoms & & & & & & & $\mathrm{x}$ \\
\hline $\begin{array}{l}\text { Symptoms commonly reported } \\
\text { by cancer patients }\end{array}$ & & $\mathrm{x}$ & & & & $\mathrm{x}$ & $\mathrm{x}$ \\
\hline
\end{tabular}

EORTC QLQ-C30 European Organisation for Research and Treatment of Cancer Quality of Life Questionnaire-Core 30, EQ-5D EuroQoL 5-Dimension, FACT-LYM Functional Assessment of Cancer Therapy-Lymphoma, FACT-G Functional Assessment of Cancer Therapy-General, MFI Multidimensional Fatigue Inventory, PRO patient-reported outcome, PROMIS-29 Patient-Reported Outcomes Measurement Information System-29, WPAI Work Productivity and Activity Impairment questionnaire

coding scheme was developed and operationalized using MAXQDA version 18.2.0 (VERBI GmbH, Berlin), a software program designed for qualitative analysis. Coding was an iterative process, and the initial code list was updated as necessary to reflect the actual terms patients used to describe concepts. The coding process was guided by established qualitative research methods, including grounded theory and constant comparative techniques [40, 41]. In grounded theory, inductive yet systematic analytic strategies are applied to qualitative data to conceptually analyze lived experiences. Unique concepts were identified and grouped into broader categories, which helped to identify and explain patterns and relationships within the data sets [42, 43]. Ultimately, frequencies of unique concept endorsements were aggregated and interpreted, with accompanying exemplary quotes.
After analysis of the transcripts was completed, the findings were compared with the PROs identified from the literature review to understand the level of patient endorsement across the instruments. High endorsement was classified as $>50 \%$ patient endorsement, moderate endorsement as $25-50 \%$ patient endorsement, and low endorsement as $<25 \%$ patient endorsement.

\section{RESULTS}

Eighteen patients from across the United States participated in two focus groups. The first focus group was conducted in person in Dallas, Texas, and included 11 patients with DLBCL from New York, Illinois, Texas, Florida, California, Georgia, and Indiana. The second focus group was conducted via Zoom with seven patients with 
Table 2 Patient demographic and health information

\begin{tabular}{|c|c|c|c|}
\hline Characteristic & $\begin{array}{l}\text { First focus group Dallas, TX } 2019 \\
(n=11)\end{array}$ & $\begin{array}{l}\text { Second focus group virtual } 2020 \\
(n=7)\end{array}$ & $\begin{array}{l}\text { Total sample } \\
(n=18)\end{array}$ \\
\hline \multicolumn{4}{|l|}{ Age (years) } \\
\hline Mean & 59 & 62 & 60 \\
\hline Minimum-maximum & $33-80$ & $46-72$ & $33-80$ \\
\hline \multicolumn{4}{|l|}{$\operatorname{Sex}(n, \%)$} \\
\hline Male & $7(63.6 \%)$ & $4(57.1 \%)$ & $11(61.1 \%)$ \\
\hline Female & $4(36.4 \%)$ & $3(42.9 \%)$ & $7(38.9 \%)$ \\
\hline \multicolumn{4}{|l|}{ Race/ethnicity ( $n, \%)$} \\
\hline White or Caucasian & $9(81.8 \%)$ & $7(100.0 \%)$ & $16(88.9 \%)$ \\
\hline Data not provided & $2(18.2 \%)$ & $0(0.0 \%)$ & $2(11.1 \%)$ \\
\hline \multicolumn{4}{|c|}{ Time since DLBCL diagnosis (years) } \\
\hline Mean & 5.1 & 9.0 & 6.6 \\
\hline Minimum-maximum & $1.3-19.8$ & $3.5-17.5$ & $1.3-19.8$ \\
\hline \multicolumn{4}{|c|}{ Time since CAR T treatment (years) } \\
\hline Mean & 1.1 & 1.9 & 1.4 \\
\hline Minimum-maximum & $0.5-1.7$ & $1.0-2.5$ & $0.5-2.5$ \\
\hline
\end{tabular}

CAR $T$ chimeric antigen receptor T-cell, DLBCL diffuse large B-cell lymphoma

DLBCL from Maryland, Idaho, California, and Texas.

\section{Patient Demographics and Characteristics}

The mean age of the patients was 60 years (range $33-80$ years). Approximately 60\% $(n=11)$ of the patients were male, and a majority of them $(n=16,88.9 \%)$ were Caucasian. The average time since DLBCL diagnosis was 6.6 years (range 1.3-19.8 years). The average time since CAR $\mathrm{T}$ therapy administration was 1.4 years (range 0.5-2.5 years). Full demographic and health information of the patients is shown in Table 2.

\section{Qualitative Results}

Eight main domain impairments were identified in the qualitative analysis: social functioning, emotional functioning, fatigue, physical functioning, cognitive functioning, role functioning, sleep, and pain/discomfort. The frequency of patients' reported domain endorsement (classified as patients who experienced impairment, patients who did not experience impairment, and patients who did not provide input, hereafter referred to as non-responders) throughout CAR T treatment is summarized in Fig. 1, and exemplary quotes are provided throughout the Results section of the domains as well as in Table 3. Results only reflect verbally expressed comments.

\section{Patient Impact}

Social Functioning Impairment of social functioning was the most frequently endorsed domain among focus group participants. Prior to CAR T therapy, 13 patients $(72 \%$ of the total sample) highlighted having difficulty 
Table 3 Patient representative quotes

Domain
Social functioning
The ability to perform everyday social tasks
appropriately and to maintain a fulfilling social life
and relationships with peers. Commonly described
by individuals' interactions with their
environment and the ability to fulfill their social
roles

Emotional functioning

The ability to regulate, recognize, and express a myriad of emotions, which is commonly conceptualized as the balance of feelings (positive and negative) experienced in life and the perceived feelings of happiness and satisfaction

Physical functioning

The ability to perform physical activities necessary for daily living and for maintaining functional independence

\section{Cognitive functioning}

The ability to perform mental processes including perception, memory, learning, attention, decisionmaking, and communication, which are typically the requirements for reasoning and acquisition of knowledge

\section{Time frame Verbatim response}

Before CAR T “You don't have much of a social life ... you're basically living in a bubble.”

During/

"I couldn't do anything ... I couldn't be around immediately [others] afterwards.” after CAR T

Six months "You can't make it to every basketball game for your after CAR T kids...go to some of the school functions because frankly counts are low.”

Before CAR T “[I felt] fear and isolated because I didn't know anyone else who was going through it.”

During/ 'I wasn't fearful that I couldn't make it through it, immediately but it's more the unknown of some of these side after CAR T effects seem pretty wicked.”

Six months "... [It's] the hardest part for me because you feel after CAR $\mathrm{T}$ like you're just getting ready to start living your life... and then all of a sudden it hits you again. And then that little voice in the back of your head, 'Is it going to come back? Is it going to come back?"

Before CAR T "I [had] gone through 8 months of terrible chemo and my body was wrecked.”

During/ "I could hardly drag around ... eventually [I] could immediately get to the bathroom on my own, but with a after CAR T walker, walked around the circuit of the [hospital] floor... it was pretty debilitating.”

Six months "[This recovery] keeps me from doing strenuous after CAR T things, like if I try to walk too much...”

Before CAR T I couldn't multitask. I couldn't-it was hard to focus on a lot of things ..."

During/ "With the CAR T, it took just a few months. So, I immediately would read something and then reread it because after CAR T I couldn't concentrate enough on it.”

Six months "... it was a couple months before I could really sort after CAR T of focus.” 
Table 3 continued

Domain
Role functioning
The ability to be involved in life situations related
to work, family life, relationship with partner,
household chores, leisure-time activities,
community involvement (including volunteer
work), and everyday activities

Sleep

The experience of negative changes in sleep quality, sleep depth, and perceived difficulties in getting to sleep or staying asleep

Fatigue

The experience of extreme tiredness resulting from mental or physical exertion

\section{Time frame Verbatim response \\ Before CAR T "I had been out [of work] for 9 months, straight sick that I couldn't do because I had been so sick with chemo and other things that" \\ During/ 'I couldn't be around students afterwards, so I kind immediately of took a leave of absence. Then I ended up after CAR T retiring."}

Six months "I work half the amount of time. I only work 2 days after CAR T a week now. and I've been slowly narrowing it down just because it takes me a full day to recover.”

Before CAR T "I just always have trouble sleeping."

During/ "I required a lot of extra sleep, at least 10 or immediately 11 hours, and I normally never slept more than after CAR T 7.”

Six months "I do wake up in the middle of the night about 3 or after CAR T 4 times a month just wide awake. It takes a couple of hours to be tired enough to fall back asleep. That has happened since CAR T.”

Before CAR T "I think it's indescribable, the tiredness, especially before like when I knew something was wrong, but I wasn't diagnosed yet, it was like a fatigue I have never experienced in my life, like being not even being able to keep my eyes open for more than a couple hours at a time.”

During/ "I remember people reminding me that for every immediately day in the hospital probably it is going to take you after CAR T a week to recover ... your stamina, muscle tone and so on was and anything that probably was true."

Six months "I know that I don't have the stamina or the energy after CAR T that I had before, but I'm not getting out and exercising as much, except walking around our neighborhood. I'm not doing any fishing or camping or hiking, or anything like that." 
Table 3 continued

\begin{tabular}{|c|c|c|}
\hline Domain & Time frame & Verbatim response \\
\hline \multirow[t]{3}{*}{$\begin{array}{l}\text { Pain/discomfort } \\
\text { A localized or generalized unpleasant bodily } \\
\text { sensation or complex of sensations that causes } \\
\text { mild to severe physical discomfort or pain }\end{array}$} & Before CAR T & $\begin{array}{l}\text { "My cancer is concentrated in my core. I had } \\
\text { hundreds of lymph nodes the size of golf balls in } \\
\text { my core and they were pressing up against } \\
\text { nerves..." }\end{array}$ \\
\hline & $\begin{array}{l}\text { During/ } \\
\text { immediately } \\
\text { after CAR T }\end{array}$ & $\begin{array}{l}\text { "I had shooting pains and I didn't have a constant } \\
\text { headache, but I did have the stabbing pain in my } \\
\text { head just out of the blue for probably just a flash, } \\
\text { and extremely painful. That started on like } \\
1 \text { week after the infusion of the T cells." }\end{array}$ \\
\hline & $\begin{array}{l}\text { Six months } \\
\quad \text { after CAR T }\end{array}$ & $\begin{array}{l}\text { "I have now like joint pain a lot and a lot of back } \\
\text { pain. I mean, I don't go a day without; } \\
\text { unfortunately I'm on pain medicine. So, that's } \\
\text { gotten a lot worse." }\end{array}$ \\
\hline
\end{tabular}

maintaining relationships with others and a lack of fulfillment in their social life. One participant described this as "You don't have much of a social life ... you're basically living in a bubble." This issue remained static for patients in the first focus group, but more patients from the second focus group endorsed social functioning impairments during and immediately after CAR T therapy $(n=7,100 \%)$, compared with only four $(57 \%)$ patients before CAR T therapy. Most notably, a total of 16 patients (89\%) from both groups experienced impairment in social functioning. Six months after CAR T therapy, six patients (33\% of the total sample) highlighted lasting limitations in social functioning, five ( $28 \%$ of the total sample) patients had no social functioning limitations, and seven (39\% of the total sample) patients were non-responders.

Emotional Functioning Difficulty with emotional functioning was another highly endorsed domain among focus group participants throughout their experience with CAR T therapy. Eleven patients ( $61 \%$ of the total sample) reported struggling with their emotional wellbeing before CAR $\mathrm{T}$ therapy. One participant commented "[I felt $]$ fear and isolated because I didn't know anyone else who was going through it." Thirteen patients ( $72 \%$ of the total sample) reported experiencing this impact during and immediately after CAR $\mathrm{T}$ therapy and cited particular emotional challenges during this time period. Patients emphasized their experience in emotional functioning during and immediately after CAR T therapy as being primarily related to concerns about efficacy of treatment and side effects like CRS and neurotoxicity syndromes. At 6 months after treatment, the number of patients who endorsed emotional impact decreased to seven $39 \%$ of the total sample) and was equivalent to the number who did not endorse it $(n=7$ [39\%]).

Physical Functioning Seven patients (39\% of the total sample) reported difficulty in physical functioning prior to CAR $\mathrm{T}$ therapy. Twelve patients (67\% of the total sample) reported impairment of physical functioning during and immediately after CAR T therapy. Only patients from the live focus group $(n=4)$ reported impairments in physical functioning at 6 months after CAR T therapy. One participant described the experience as "I could hardly drag around ... eventually [I] could get to the bathroom 
Table 4 Level of endorsement of common domains reported by DLBCL patients treated with CAR T therapy

\begin{tabular}{llllllll}
\hline Conceptual domain & \multicolumn{2}{l}{ Degree of endorsement } & & & & \\
\cline { 2 - 7 } & EORTC QLQ-C30 & EQ-5D & WPAI & PROMIS-29 & MFI & FACT-G & FACT-LYM $^{\mathbf{c}}$ \\
\hline Physical functioning & High $^{\mathrm{a}}$ & High & NA $^{\mathrm{b}}$ & High & NA & High & High \\
Emotional functioning & High & High & NA & High & NA & High & High \\
Fatigue & High & NA & NA & NA & High & High & High \\
Social functioning & High & NA & NA & High & NA & High & High \\
Role functioning & High & NA & High & NA & NA & NA & NA \\
Cognitive functioning & Moderate & NA & NA & NA & NA & NA & NA \\
Pain/discomfort & Moderate & Moderate & NA & Moderate & NA & Moderate & Moderate \\
Sleep & Moderate & NA & NA & Moderate & NA & NA & NA \\
General symptoms & Moderate & NA & NA & NA & NA & Moderate & Moderate
\end{tabular}

CAR T chimeric antigen receptor T-cell, $D L B C L$ diffuse large B-cell lymphoma, EORTC QLQ-C30 European Organisation for Research and Treatment of Cancer Quality of Life Questionnaire-Core 30, EQ-5D EuroQoL 5-Dimension, FACTLYM Functional Assessment of Cancer Therapy-Lymphoma, FACT-G Functional Assessment of Cancer Therapy-General, MFI Multidimensional Fatigue Inventory, PRO patient-reported outcome, PROMIS-29 Patient-Reported Outcomes Measurement Information System-29, WPAI Work Productivity and Activity Impairment

${ }^{a} \mathrm{High}$ endorsement was classified as $>50 \%$ patient endorsement, moderate endorsement as $25-50 \%$ patient endorsement, and low endorsement as $<25 \%$ patient endorsement

${ }^{\mathrm{b}} \mathrm{NA}=$ Not applicable, as PRO did not have the domain

'The lymphoma-specific symptom domain of the FACT-LYM was not included in this analysis

on my own, but with a walker, walked around the circuit of the [hospital] floor... it was pretty debilitating." Most patients also noted that the lasting impairments were less severe than those experienced before or during CAR T therapy. Additionally, seven patients $(39 \%$ of the total sample) reported no impairment, and seven patients (39\% of the total sample) were also non-responders.

Cognitive and role functioning A small portion of patients highlighted impairments of cognitive functioning and role functioning before CAR T therapy ( $n=5$ [28\%] for each). A larger number of patients reported impairment of both domains during and immediately following CAR T therapy. Patients with cognitive functioning impairments during and immediately following CAR $\mathrm{T}$ therapy $(n=10$ [56\%]) commonly cited neurological adverse events like delirium, confusion, and seizures as the cause of the impairment of cognitive functioning. One patient provided descriptions such as "I had been out [of work] for 9 months straight sick that I couldn't do because I had been so sick with chemo and other things that" and "I couldn't multitask. I couldn't-it was hard to focus on a lot of things ..." Similarly, several patients cited an inability or limitation to their work and daily roles during CAR $\mathrm{T}$ therapy and in the immediate recovery period ( $n=13$ [72\%]). After 6 months, one patient ( $6 \%$ of the total sample) reported cognitive limitations, eight patients (44\%) did not experience impairment, and the remaining $50 \%(n=9)$ of the total sample were non-responders. The number of patients who experienced role functioning impairment returned to the same number before CAR $\mathrm{T}$ therapy ( $n=5$ of the total sample [28\%]), and a larger percentage $(50 \%)$ of patients reported not experiencing impairment in role functioning at 6 months after CAR T. 
Sleep Sleep impairment was reported by some patients before $(n=4$ [22\%]), during and immediately after $(n=8 \quad[44 \%])$, and at 6 months after ( $n=6$ [33\%]) CAR T therapy, but the impairment was not reported to be particularly severe or bothersome by the patients. In all three time points, a large number of nonresponders was observed $(n=10 ; 56 \%$ of the total sample both before and 6 months after CAR T therapy).

\section{Fatigue and Pain/Discomfort}

Both mental and physical aspects of fatigue were frequently discussed by patients when characterizing their experiences with DLBCL and CAR T therapy. Patients struggled to isolate the cause of their fatigue because their prior DLBCL treatments left them with reduced energy levels. Preexisting fatigue was experienced by nine patients (50\% of the total sample) prior to CAR T therapy. During and immediately following CAR T therapy, 13 patients (72\% of the total sample) noted new onset or increased intensity of fatigue. At 6 months after CAR T therapy, 10 patients $(56 \%$ of the total sample) reported experiencing fatigue; however, compared with the intensity of fatigue experienced during and immediately after CAR $\mathrm{T}$ therapy, the consensus was that energy levels continued to gradually improve in the months following CAR T therapy.

Only one patient (6\%) reported experiencing preexisting pain prior to CAR T therapy, which was attributed to nerve damage as a result of several rounds of chemotherapy. During and immediately following CAR $\mathrm{T}$ therapy, six additional patients reported experiencing pain $(n=7$ [39\%]). All patients who experienced severe headache during and immediately following CAR $\mathrm{T}$ therapy noted that it resolved within a few weeks; however, other types of pain/discomfort (back pain, joint pain, muscle cramps) lingered. At 6 months after CAR $T$ therapy, eight patients ( $44 \%$ of the total sample) reported persistent pain. Although over $50 \%$ of patients were non-responders before and 6 months after CAR $\mathrm{T}$ as it relates to pain/discomfort, the number of patients who experienced impairment after CAR $\mathrm{T}$ increased as compared with before CAR $\mathrm{T}$.

\section{Caregiver Burden}

No additional patient-related domains emerged from the concept confirmation portion of the interviews. When asked to share additional thoughts on the experience of DLBCL and CAR $\mathrm{T}$ therapy, patients commented on the perceived caregiver burden faced by their partners or family members. All patients reported that they had caregivers to assist them during the CAR $T$ therapy process and one patient remarked, "...cancer is harder on the people that love you than it is on you because you're just so focused on what's happening to your body at the time and trying to get through the day, but they're totally with it and their minds are spinning all time. I think it is much harder." Other patients noted that caregivers assumed several care management responsibilities like coordinating appointments and making key treatment decisions that negatively impacted the caregiver on a personal or professional level. One patient commented, "[My wife] really had to drop everything and take care of me all summer last year. Which she was happy to do, and she did an amazing job taking care of me. ... It was just an incredibly stressful time for her. It certainly cost her her career, in terms of interrupting her important research. It's really tough on caregivers. She works full time, it's a very intense job, has a lot of responsibilities. It's been really hard."

\section{Domain Endorsement}

In the focus group discussions, patients endorsed and confirmed all the identified domains from the literature review. Results from this analysis are shown in Table 4, with the degree of endorsement of the domains: low endorsement, moderate endorsement, and high endorsement. The EORTC QLC-30 has the most comprehensive set of domains that were all highly (physical functioning, emotional functioning, fatigue, social functioning, role functioning/productivity) or moderately (cognitive functioning, pain/discomfort, sleep impairments, general symptoms) endorsed by the DLBCL population in this study. Similarly, the FACT-G, FACT-LYM, and PROMIS-29 also covered highly and moderately relevant domains 
endorsed by the sample. All PROs assessed contained highly relevant domains for the DLBCL sample treated with CAR T.

\section{DISCUSSION}

Nearly all patients in this study experienced a decrease in their HRQoL before CAR $\mathrm{T}$ treatment. Since R/R DLBCL patients who are suitable candidates for CAR $\mathrm{T}$ therapy have generally exhausted multiple lines of treatment, these patients may already have HRQoL deficits resulting from the disease itself and prior treatment [44]. Additionally, some patients were unable to distinguish impairment attributed to their condition from previous treatment or from CAR $\mathrm{T}$ treatment. Various studies have identified impairment of HRQoL in DLBCL patients, especially in physical and functional well-being after the first-line treatment [24-26, 45-47]. Due to the toxicities associated with CAR $\mathrm{T}$ therapy and limited treatment options, it was not surprising that more patients reported impairment across all domains during CAR T treatment compared with before CAR T treatment.

Sidana et al. [48] reported a worsening of HRQoL, specifically for functional and physical well-being, immediately after CAR T treatment, but an improvement was observed at 3 months after treatment. Similarly, across all domains in this study except for pain/discomfort, fewer patients reported impairment at 6 months after CAR T compared with immediately after CAR T treatment. Additionally, fewer patients reported impairments in social functioning, emotional functioning, physical functioning, or cognitive functioning at 6 months after CAR T compared with before CAR $\mathrm{T}$ treatment. Unlike findings from Sidana et al. [48], who reported little change in social and family well-being, the patients in our study reported that social functioning was highly impacted throughout their CAR T treatment.

Given the known neurological side effect potential of CAR T therapy, it was not surprising that patients in our study reported an increase in cognitive impairment during treatment and the need to limit or stop working. However, only one person reported cognitive impairment at 6 months after CAR T therapy. While longterm cognitive deficits due to CAR T therapy have been raised as a concern [44], the findings from this study echo those of Ruark et al. [49], who also noted that overall self-reported neuropsychiatric outcomes such as low rates of anxiety, depression, and social function in survivors after CAR $\mathrm{T}$ therapy showed no clinically meaningful difference from the general US population. Furthermore, the patient in our study who reported cognitive deficits had trouble attributing these deficits to age or treatment; this concept may be difficult to assess, as some older patients may be at higher risk of cognitive dysfunction [44].

Not surprisingly, emotional functioning was highly endorsed. In addition to the anxiety or depression that patients might feel, especially in the early stages of treatment, the emotional impact such as isolation and fear associated with a novel therapy, including not knowing anyone who has undergone this treatment, is an added burden and may be somewhat unique and likened to a rare disease [50]. Most notably, more patients in our study reported symptoms of fatigue and pain/discomfort after CAR T than before CAR T therapy. Several patients reported their fatigue continued for 6 months after treatment, although the sleep impairments reported in this study were not considered particularly bothersome. Our results are consistent with results from Price et al. [51], who also detected concerns about sleep and energy levels in a nationwide survey with DLBCL patients. Yanez et al. [52] reported that fatigue associated with CAR $\mathrm{T}$ therapy generally resolves within 3 weeks after infusion, which suggests that this symptom may have resolved earlier than the time frame investigated in this study.

Most notably, in the current study, all patients reported having caregivers who were affected by their condition and CAR T therapy. Caregivers play a vital role in the well-being of patients undergoing CAR T, and their HRQoL should also be taken into consideration when evaluating treatment outcomes [44]. A previous study in patients treated with HSCT reported poor HRQoL for one in five caregivers compared with the general population and an increase in 
depression and sleep disorders [53]. Future studies evaluating caregivers' HRQoL, especially when major toxicities are anticipated during CAR T therapy, may help to identify areas where interventions could decrease their burden $[19,44,54]$. With the growing interest in measuring caregivers' HRQOL and integrative oncology programs, there is a unique opportunity to provide better support for caregivers and patients.

The endorsement of the domains for commonly used PROs to assess DLBCL patients was high. Cognitive functioning, pain/discomfort, sleep impairments, and general symptoms were moderately endorsed. The EORTC QLQ-30, which contains the highest number of domains among the PROs, was highly or moderately endorsed. It also demonstrated statistically significant or clinically meaningful changes in a greater number of domains compared with the FACT-Lym [26]. Currently, the EORTC is developing and validating the QLQ-C30 for patients with high-grade/low-grade NHL [55]. This validation process is ongoing, and data collection is expected to end in 2021, with the analyses and report to follow. While most concepts evaluated in this study were adequately represented in PROs that covered HRQoL and disease symptomology, it is possible that a CAR T-specific measure would be needed to assess the impact of treatment-related toxicities such as CRS and neurotoxicity side effects. However, the overall findings provide further evidence supporting the concepts included in these PROs for this population.

Limitations of this study are primarily associated with the patient sample. These patients were commercially treated and participated in both an advisory board panel and focus group. The patient selection in this setting may be inherently biased, as it was a highly motivated group that responded to treatment. Given the length of time from the treatment, the sample is also likely to have a high proportion of patients with a desirable efficacy outcome; those with deleterious outcomes may report differently. Patients in the first focus group had the stamina and well-being to travel onsite. Therefore, we are unable to extrapolate results of this study to patient experiences for whom treatment was not successful or for those that relapsed. Additionally, patients in this study were predominantly Caucasian English speakers, and future studies should include other ethnicities to assess any differences in how these patients may experience symptom burden and impact on HRQoL. Secondly, only domain-level coverage of the identified PROs was evaluated, and nuances of evaluating the PROs at the item level were not conducted for this study. However, the purpose of this study was to assess whether the concepts of the identified PROs were appropriately relevant and to explore whether there were any gaps or missing concepts, rather than instrument validation. Another limitation of this study is that patients were asked to recall their experiences from an average of 1.4 years ago and in some patients from up to 2.5 years ago. While the elapsed time could make it more likely that patients individually reported on experiences that were highly relevant, the reliance on patient memory introduces recall bias, with longer recall periods associated with increased bias. Lastly, while live focus groups are preferred, the platform for the second focus group was virtual due to the COVID-19 pandemic. To some extent, this may have affected the amount of participation observed between the two groups. Although trends in lack of participation were not observed with either group, the virtual group attendees appeared more eager to participate, which may be attributed to a desire for social interaction during stay-at-home orders. However, there were patients who did not respond for all domains in both focus groups, which is commonly observed in qualitative research. It is important to note that a non-response cannot be interpreted as not having experienced an impact in the domain.

Despite these limitations, this study provides important new information on DLBCL patients' experiences with CAR T treatment. Qualitative research, such as this, provides direct patient input and perspectives, which may help to ensure that studies are measuring what really matters to patients. 


\section{CONCLUSIONS}

This study suggests that the current PRO domains commonly used to assess DLBCL are appropriate for assessing patients treated with CAR T. Identifying and understanding the relevance of these domains for CAR T patients can help clinicians better understand this population's needs and treatment journey and help inform the selection and use of PROs in future studies, in particular HRQoL for caregivers and CAR T-specific HRQoL measures, which may address ICANS and CRS. Additionally, these data can be used to help inform programs that may provide better support to DLBCL patients and caregivers.

\section{ACKNOWLEDGEMENTS}

The authors would like to express their gratitude to the patients for their collaboration in this research and willingness to discuss their experiences with DLBCL and CAR T therapy.

Funding. Funding for this research, including the journal's Rapid Service Fees, was provided by Kite.

Medical Writing, Editorial, and Other Assistance. Editorial and writing assistance in the preparation of this article was provided by Rebecca Cheng and Kayla Scippa; who are former employees of ZS and were employed by ZS at the time of research, Nils Fischer, Larisa Gofman, and Aparna Sharma of ZS Associates. Sally Wade of Wade Outcomes provided project management and editorial support.

Authorship. All named authors meet the International Committee of Medical Journal Editors (ICMJE) criteria for authorship for this article, take responsibility for the integrity of the work, and have given their approval for this version to be published.

Author Contributions. All named authors contributed to the study conception and design. Rebecca Cheng and Kayla Scippa oversaw data collection and analysis; all authors reviewed study results. Julia Thornton Snider, Kayla Scippa, and Rebecca Cheng collaborated to draft the manuscript. All named authors made substantive revisions to the draft manuscript, and all read and approved the final manuscript.

Disclosures. Rebecca Cheng was employed by ZS Associates at the time this study was conducted. Kayla Scippa was employed by ZS Associates at the time this study was conducted and has consulted at Parexel International. Frederick Locke has received research funding from Kite, a Gilead Company, Allogene, and Novartis, and has also consulted to ecoR1, Emerging Therapy Solutions Gerson Lehman Group, Allogene, Amgen, Bluebird Bio, BMS/Celegene, Calibr, Iovance,Kite, Janssen, Legend Biotech, Novartis, Umoja, Cowen, Cellular Biomedicine Group, GammaDelta Therapeutics, and Wugen. Several patents are held by his institution in his name (unlicensed) in the field for cellular immunotherapy. Julia Thornton Snider is an employee and stockholder of Kite, a Gilead Company. Heather Jim has received research funding from Kite, a Gilead Company, and consulted to Janssen Scientific Affairs and Merck.

Compliance with Ethics Guidelines. The New England IRB reviewed all study documents and provided initial approval for the study in November 2019. The study materials were amended in the early 2020 to accommodate conduct of the second focus group virtually on account of COVID-19 pandemic and statewide variances in reopening plans and travel restrictions. The authors have read and understood the declaration intended by the declaration of Helsinki as a statement of ethical principles for medical researching involving human subjects, including research on identifiable human data. The study was performed in accordance with the Helsinki Declaration of 1964 , and its later amendments. The IRB review was finalized in July 2020. All patients completed an informed consent form (ICF) indicating their willingness to participate in this research. All patients indicated their willingness to allow publication 
of study findings as part of their ICF. The datasets generated during and/or analyzed during the current study are available from the corresponding author on reasonable request. In addition to consenting to the study, the consent form also included a information on how results may be used in a publication: "If the results of the study are published, your identity will remain confidential. Your name will not be used in any study reports. All study reports will be used for research purposes only. To protect your privacy, your responses will be labeled with only a subject ID and first name as an identifier. Your responses and data may be transmitted to state/provinces and other countries to help conduct this study, including checking the accuracy of the data collected and overseeing the conduct of the study." Each patient consented to all parameters of the consent form prior to each interview.

Data Availability. Data sharing is not applicable to this article as no datasets were generated or analyzed during the current study.

Open Access. This article is licensed under a Creative Commons Attribution-NonCommercial 4.0 International License, which permits any non-commercial use, sharing, adaptation, distribution and reproduction in any medium or format, as long as you give appropriate credit to the original author(s) and the source, provide a link to the Creative Commons licence, and indicate if changes were made. The images or other third party material in this article are included in the article's Creative Commons licence, unless indicated otherwise in a credit line to the material. If material is not included in the article's Creative Commons licence and your intended use is not permitted by statutory regulation or exceeds the permitted use, you will need to obtain permission directly from the copyright holder. To view a copy of this licence, visit http:// creativecommons.org/licenses/by-nc/4.0/.

\section{APPENDIX}

The EuroQol Five Dimension (EQ-5D) is a fiveitem instrument that uses self-reported mobility, self-care, usual activities, pain/discomfort, anxiety/depression to assess generic health-related quality of life [56]. The EQ-5D has been validated and used in a multiple languages, diverse populations, and wide range of disease areas including oncology $[56,57]$.

The European Organisation for the Research and Treatment of Cancer Quality of Life Questionnaire (EORTC QLQ-C30) is a 30-item disease-specific instrument that uses five functional scales (physical, role, cognitive, emotional, social); three symptom scales (fatigue, pain, nausea and vomiting); one global health status/quality-of-life scale, and single items assessing additional symptoms commonly reported by cancer patients (dyspnea, loss of appetite, insomnia, constipation, diarrhea) as well as the perceived financial impact of the disease to evaluate the quality of life of cancer patients [32]. The EORTC QLQ-C30 has been translated into and validated in over 100 languages, and is used in more than 5000 research studies each year [58].

The Work Productivity and Activity Impairment (WPAI) assessment uses six self-report items to collect data on work absenteeism, productivity loss, presenteeism, and impairment of work and regular activities due to health problems [38]. The WPAI has been validated in a variety of populations and disease areas[59], and numerous translations are available. The WPAI developers offer a generic health version (WPAI:GH) and a version that may be used to assess the impact of specific health problems (WPAI:SHP).

The Patient-Reported Outcomes Measurement Information System (PROMIS) is a stateof-the-science self-report tool developed under the auspices of the National Institutes of Health to assess patient-reported outcomes in the clinical research setting [60]. The PROMIS-29 is a validated instrument that uses a 0-10 scale to assess pain intensity, and also assesses seven health domains (physical function, fatigue, pain interference, depressive symptoms, 
anxiety, ability to participate in social roles and activities, sleep disturbance) using four items for each domain $[61,62]$.

The Multidimensional Fatigue Inventory (MFI) uses 20 self-report times to assess five dimensions: general fatigue, physical fatigue, mental fatigue, reduced motivation, and reduced activity [35]. The MFI has been validated in a variety of populations including cancer patients receiving radiotherapy, patients with the chronic fatigue syndrome, psychology students, medical students, army recruits, and junior physicians $[35,63]$.

The Functional Assessment of Cancer Treatment-General (FACT-G) is a validated instrument that uses 27 self-report items to assess four quality-of-life domains (physical, social/family, emotional, functional well-being) in patients receiving cancer treatment $[33,64]$. The FACT$\mathrm{G}$ has been used to assess QoL in patients with various tumor types and has been translated into numerous languages [64].

The Functional Assessment of Cancer Treatment-Lymphoma (FACT-LYM) includes the items in the FACT-G along with a 15-item lymphoma-specific subscale that assesses common symptoms related to lymphoma and/or its treatment (e.g., pain, fever, swelling, night sweats, insomnia, itching, weight loss, fatigue, loss of appetite) [65]. The FACT-LYM has been validated and translations are available from the developer [34].

\section{REFERENCES}

1. Hunt KE, Reichard KK. Diffuse large B-cell lymphoma. Arch Pathol Lab Med. 2008;132:118-24.

2. Teras LR, DeSantis CE, Cerhan JR, Morton LM, Jemal A, Flowers CR. 2016 US lymphoid malignancy statistics by World Health Organization subtypes. CA Cancer J Clin. 2016;66:443-59.

3. Tilly H, da Silva MG, Vitolo U, et al. Diffuse large B-cell lymphoma (DLBCL): ESMO clinical practice guidelines for diagnosis, treatment and follow-up. Ann Oncol. 2015;26(suppl 5):v116-25.

4. SEER Cancer Statistics Review (CSR) 1975-2017. National Cancer Institute-National Institute of Health. https://seer.cancer.gov/statfacts/html/ dlbcl.html. Accessed Jan 2021.
5. Liu Y, Barta SK. Diffuse large B-cell lymphoma: 2019 update on diagnosis, risk stratification, and treatment. Am J Hematol. 2019;94:604-16.

6. National Comprehensive Cancer Network ${ }^{\circledR}$ NCCN Clinical Practice Guidelines in Oncology (NCCN Guidelines) ${ }^{\circledR}$. B-Cell Lymphomas. Version 4.2021. https://www.nccn.org/professionals/physician_gls/ pdf/b-cell.pdf.

7. Ollila TA, Olszewski AJ. Extranodal diffuse large B cell lymphoma: molecular features, prognosis, and risk of central nervous system recurrence. Curr Treat Option Oncol. 2018;19:38.

8. Zelenetz AD, Gordon LI, Abramson JS, et al. NCCN guidelines insights: B-cell lymphomas, version 3 . 2019. J Natl Compr Canc Netw. 2019;17:650-61.

9. Chiappella A, Santambrogio E, Castellino A, Nicolosi M, Vitolo U. Integrating novel drugs to chemoimmunotherapy in diffuse large B-cell lymphoma. Expert Rev Hematol. 2017;10:697-705.

10. Friedberg JW. Relapsed/refractory diffuse large B-cell lymphoma. Hematol Am Soc Hematol Educ Program. 2011;2011:498-505.

11. Crump M, Neelapu SS, Farooq U, et al. Outcomes in refractory diffuse large B-cell lymphoma: results from the international SCHOLAR-1 study. Blood. 2017;130:1800-8.

12. Nagle SJ, Woo K, Schuster SJ, et al. Outcomes of patients with relapsed/refractory diffuse large B-cell lymphoma with progression of lymphoma after autologous stem cell transplantation in the rituximab era. Am J Hematol. 2013;88:890-4.

13. Philip T, Guglielmi C, Hagenbeek A, et al. Autologous bone marrow transplantation as compared with salvage chemotherapy in relapses of chemotherapy-sensitive non-Hodgkin's lymphoma. N Engl J Med. 1995;333:1540-5.

14. Telio D, Fernandes K, Ma C, et al. Salvage chemotherapy and autologous stem cell transplant in primary refractory diffuse large B-cell lymphoma: outcomes and prognostic factors. Leuk Lymphoma. 2012;53:836-41.

15. Brudno JN, Kochenderfer JN. Chimeric antigen receptor T-cell therapies for lymphoma. Nat Rev Clin Oncol. 2018;15:31-46.

16. Neelapu SS, Locke FL, Bartlett NL, et al. Axicabtagene ciloleucel CAR T-cell therapy in refractory large B-cell lymphoma. N Engl J Med. 2017;377:2531-44.

17. Schuster SJ, Bishop MR, Tam CS, et al. Tisagenlecleucel in adult relapsed or refractory diffuse large B-cell lymphoma. N Engl J Med. 2019;380:45-56. 
18. Abramson JS, Palomba ML, Gordon LI, et al. Lisocabtagene maraleucel for patients with relapsed or refractory large B-cell lymphomas (TRANSCEND NHL 001): a multicentre seamless design study. Lancet. 2020;396:839-52.

19. Lopez G, Lacey J, Christie AJ, et al. Patient-reported outcomes in integrative oncology: bridging clinical care with research. Cancer J. 2019;25:311-5.

20. Nipp RD, Temel JS. Harnessing the power of patient-reported outcomes in oncology. Clin Cancer Res. 2018;24:1777-9.

21. Warsame R, D'Souza A. Patient reported outcomes have arrived: a practical overview for clinicians in using patient reported outcomes in oncology. Mayo Clin Proc. 2019;94:2291-301.

22. Zagadailov E, Fine M, Shields A. Patient-reported outcomes are changing the landscape in oncology care: challenges and opportunities for payers. Am Health Drug Benefits. 2013;6:264-74.

23. Food and Drug Administration. Guidance for industry patient-reported outcome measures: use in medical product development to support labeling claims. 2009. p. 1-35.

24. Kelly JL, Pandya C, Friedberg JW, Mohile SG. Health-related quality of life in older patients following diffuse large B-cell lymphoma (DLBCL) diagnosis [abstract]. Blood. 2012;120:Abstract 4287.

25. Lenz G, Rutherford SC, Davies A, et al. The burden of relapsed/refractory (R/R) diffuse large B-cell lymphoma (DLBCL): a systematic literature review (SLR) [poster]. Blood. 2018;132(suppl 1):2241.

26. Lin V, Oak B, Snider J, Epstein J. Health-related quality of life (HRQOL) burden in patients with relapsed/refractory diffuse large B-cell lymphoma (RR-DLBCL) and non-Hodgkin's lymphoma (RRNHL) [abstract]. J Clin Oncol. 2020;38:Abstract e20070.

27. Lee DW, Santomasso BD, Locke FL, et al. ASTCT consensus grading for cytokine release syndrome and neurologic toxicity associated with immune effector cells. Biol Blood Marrow Transplant. 2019;25:625-38.

28. Neelapu SS, Tummala S, Kebriaei P, et al. Chimeric antigen receptor T-cell therapy-assessment and management of toxicities. Nat Rev Clin Oncol. 2018;15:47-62.

29. Gnanasakthy A, DeMuro C, Clark M, Haydysch E, Ma E, Bonthapally V. Patient-reported outcomes labeling for products approved by the Office of Hematology and Oncology Products of the US Food and Drug Administration (2010-2014). J Clin Oncol. 2016;34:1928-34.

30. Kim J, Singh H, Ayalew K, et al. Use of PRO measures to inform tolerability in oncology trials: implications for clinical review, IND safety reporting, and clinical site inspections. Clin Cancer Res. 2018;24:1780-4.

31. Food and Drug Administration. Patient-focused drug development: collecting comprehensive and representative input guidance for industry, food and drug administration staff, and other stakeholders [Final guidance]. 2020.

32. Aaronson NK, Ahmedzai S, Bergman B, et al. The European Organization for Research and Treatment of Cancer QLQ-C30: a quality-of-life instrument for use in international clinical trials in oncology. J Natl Cancer Inst. 1993;85:365-76.

33. Cella DF, Tulsky DS, Gray G, et al. The functional assessment of cancer therapy scale: development and validation of the general measure. J Clin Oncol. 1993;11:570-9.

34. Hlubocky FJ, Webster K, Beaumont J, et al. A preliminary study of a health related quality of life assessment of priority symptoms in advanced lymphoma: the National Comprehensive Cancer Network-Functional Assessment of Cancer TherapyLymphoma Symptom Index. Leuk Lymphoma. 2013;54:1942-6.

35. Smets EM, Garssen B, Bonke B, De Haes JC. The Multidimensional Fatigue Inventory (MFI) psychometric qualities of an instrument to assess fatigue. J Psychosom Res. 1995;39:315-25.

36. Rabin R, de Charro F. EQ-5D: a measure of health status from the EuroQol Group. Ann Med. 2001;33: 337-43.

37. Cella D, Riley W, Stone A, et al. The Patient-Reported Outcomes Measurement Information System (PROMIS) developed and tested its first wave of adult self-reported health outcome item banks: 2005-2008. J Clin Epidemiol. 2010;63:1179-94.

38. Reilly MC, Zbrozek AS, Dukes EM. The validity and reproducibility of a work productivity and activity impairment instrument. Pharmacoeconomics. 1993;4:353-65.

39. Grigor EJM, Fergusson D, Kekre N, et al. Risks and benefits of chimeric antigen receptor T-cell (CAR-T) therapy in cancer: a systematic review and metaanalysis. Transfus Med Rev. 2019;33:98-110.

40. Charmaz K, Smith J, Harré R, Van Langenhove L. Grounded theory. Rethinking methods in psychology. London: Sage Publications; 1995. p. 27-49. 
41. Glaser B, Strauss A. The discovery of grounded theory: strategies for qualitative research. The discovery of grounded theory. New York: Aldine de Gruyter; 1967. p. 1-18.

42. Glaser BG, Strauss AL. The constant comparative method of qualitative analysis. The discovery of grounded theory: strategies for qualitative research. New York: Aldine de Gruyter; 1967. p. 101-15.

43. Lasch KE, Marquis P, Vigneux M, et al. PRO development: rigorous qualitative research as the crucial foundation. Qual Life Res. 2010;19:1087-96.

44. Chakraborty R, Sidana S, Shah GL, Scordo M, Hamilton BK, Majhail NS. Patient-reported outcomes with chimeric antigen receptor $\mathrm{T}$ cell therapy: challenges and opportunities. Biol Blood Marrow Transplant. 2019;25:e155-62.

45. Tholstrup D, Brown PDN, Jurlander J, Jeppesen PB, Groenvold M. Quality of life in patients with diffuse large B-cell lymphoma treated with dose-dense chemotherapy is only affected temporarily. Leuk Lymphoma. 2011;52:400-8.

46. Thompson CA, Yost KJ, Maurer MJ, et al. Quality of life at diagnosis predicts overall survival in patients with aggressive lymphoma. Hematol Oncol. 2018;36:749-56.

47. van der Poel MW, Oerlemans S, Schouten HC, et al. Quality of life more impaired in younger than in older diffuse large B cell lymphoma survivors compared to a normative population: a study from the population-based PROFILES registry. Ann Hematol. 2014;93:811-9.

48. Sidana S, Thanarajasingam G, Griffin J, et al. Patient experience of chimeric antigen receptor (CAR)-T cell therapy vs. stem cell transplant: longitudinal patient reported adverse events, cognition and quality of life. Blood. 2019;134(suppl 1):794.

49. Ruark J, Mullane E, Cleary N, et al. Patient-reported neuropsychiatric outcomes of long-term survivors after chimeric antigen receptor T cell therapy. Biol Blood Marrow Transplant. 2020;26:34-43.

50. von der Lippe C, Diesen PS, Feragen KB. Living with a rare disorder: a systematic review of the qualitative literature. Mol Genet Genomic Med. 2017;5: 758-73.

51. Price M, Ravelo A, Sae-Hau M, et al. Patient-reported disease burden in chronic lymphocytic leukemia, diffuse large B-cell lymphoma, and follicular lymphoma: results from a national patient advocacy survey. J Clin Oncol. 2019;371(suppl):e18198.
52. Yáñez L, Sánchez-Escamilla M, Perales MA. CAR T cell toxicity: current management and future directions. Hemasphere. 2019;3:e186.

53. Jamani K, Onstad LE, Bar M, et al. Quality of life of caregivers of hematopoietic cell transplant recipients. Biol Blood Marrow Transplant. 2018;24: 2271-6.

54. Pratt-Chapman M, Bhadelia A. Patient-reported outcomes in health economic decision-making: a changing landscape in oncology. Recent Results Cancer Res. 2019;213:67-83.

55. Van de Poll-Franse L, et al. International development of four EORTC disease-specific quality of life questionnaires for patients with Hodgkin lymphoma, high- and low-grade non-Hodgkin lymphoma and chronic lymphocytic leukaemia. Qual Life Res. 2018;27(2):333-5.

56. Euroquol.EQ-5D. About EQ-5D. https://euroqol. org/eq-5d-instruments/. Accessed Aug 27, 2021.

57. Schwenkglenks M, Matter-Walstra K. Is the EQ-5D suitable for use in oncology? An overview of the literature and recent developments. Expert Rev Pharmacoecon Outcomes Res. 2016;16(2):207-19.

58. EORTC. Quality of Life. https://qol.eortc.org/. Accessed Aug 27, 2021.

59. WPAI References. http://www.reillyassociates.net/ WPAI_References5.html. Accessed Aug 27, 2021.

60. National Institutes of Health. Office of strategic coordination-the common fund. Patient-reported outcomes measurement information system (PROMIS). https://commonfund.nih.gov/promis/index. Accessed Aug 27, 2021.

61. Cella D, et al. PROMIS ${ }^{\circledR}$ adult health profiles: efficient short-form measures of seven health domains. Value Health. 2019;22(5):537-44.

62. Hays RD, et al. PROMIS ${ }^{\circledR}-29$ v2.0 profile physical and mental health summary scores. Qual Life Res. 2018;27(7):1885-91.

63. Smets EM, et al. Application of the multidimensional fatigue inventory (MFI-20) in cancer patients receiving radiotherapy. Br J Cancer. 1996;73(2): 241-5.

64. FACIT. FACT-G. Functional assessment of cancer therapy. https://www.facit.org/measures/FACT-G. Accessed Aug 27, 2021.

65. FACT-Lym Survey. https://8beeac51-650b-405c97a4-0987e05a41f1.filesusr.com/ugd/626819_ Odf9df363a394774af45e4cabff63ffc.pdf. Accessed Aug 27, 2021. 